\title{
A CHARACTERIZATION OF SPACES WITH VANISHING GENERALIZED HIGHER WHITEHEAD PRODUCTS
}

\author{
BY F. D. WILLIAMS ${ }^{1}$
}

\section{Communicated by R. H. Bing, November 27, 1967}

A subject of recent investigation in homotopy theory has been the study of generalized higher order Whitehead products, cf. [1] and [3]. Let us say that a space, $X$, has property $P_{n}$ if for any $f_{1}, \cdots, f_{n}$, $f_{i}: S A_{i} \rightarrow X$, we have $0 \in\left[f_{1}, \cdots, f_{n}\right]$, where $\left[f_{1}, \cdots, f_{n}\right]$ denotes the set of all $n$th order Whitehead products of $f_{1}, \cdots, f_{n}$, as defined in [3]. Thus $0 \in\left[f_{1}, \cdots, f_{n}\right]$ means that $f_{1} \vee \cdots \vee f_{n}: S A_{1} \vee \cdots$ $\vee S_{A_{n} \rightarrow X}$ can be extended to some $F: S A_{1} \times \cdots \times S A_{n} \rightarrow X$. (We note at this point that it is an unresolved conjecture as to whether $X$ has property $P_{n}$ implies that 0 is the only element of $\left[f_{1}, \cdots, f_{n}\right]$.) Now if $X$ is an $H$-space, then $X$ possesses property $P_{n}$ for all $n$, [3]. Thus multiplicative properties of $X$ itself are too strong to distinguish among the various properties $P_{n}$. On the other hand, it follows from results of [1] and [4] that a space has property $P_{2}$ if and only if its loop space is homotopy-commutative. In Theorem 1 below, we shall extend this result to characterize those spaces which have property $P_{n}$ in terms of higher homotopy-commutativity properties of their loop spaces. Since we shall wish to be able on occasion to replace a ${ }^{\circ} \mathrm{p}$ space by an equivalent $\mathrm{CW}$-monoid, we shall restrict our attention to the category of countable $\mathrm{CW}$-complexes.

The higher homotopy-commutativity properties we need are Dribed in the following definition which was introduced in [7].

vided (1) that there exist maps $Q_{i}: C(i-1) \times Y^{i} \rightarrow Y, 1 \leqq i \leqq n$, such that: (1) $Q_{1}: C(0) \times Y \rightarrow Y$ is the identity;

(2) $Q_{i}\left([\mu, \nu] \circ d_{p}(r, s), y_{1}, \cdots, y_{i}\right)=Q_{p}\left(r, y_{\mu(1)}, \cdots, y_{\mu(p)}\right)$ $\left.r \in C(p-1), \cdots, y_{v(q)}\right)$, for $(p, q)$-shuffles $(\mu, \nu)$, where $p+q=i$,

(3) if $e$ denotes the identity of $Y$, and if $y_{i}=e$, then

$$
Q_{i}\left(T, y_{1}, \cdots, y_{i}\right)=Q_{i-1}\left(D_{j}(T), y_{1}, \cdots, y_{j}, \cdots, y_{i}\right) \text {. }
$$

hull $C(i)$ is the convex linear cell described in [2], namely the convex coordine orbit of the point $(1, \cdots, n+1)$ under permutation of the $d_{p}\left(x_{1}, \cdots, x_{p}, y_{1}, \cdots, y_{i-p+1}\right)=\left(x_{1}, \cdots, x_{p}, y_{1}+p, \cdots, y_{i-p+1}+p\right)$,
${ }^{1} S_{\text {upported by the National Science Foundation Grant GP-6318. }}$ 
the map $D_{j}: C(i) \rightarrow C(i-1)$ is as defined in [2], and $[\mu, \nu]: C(i) \rightarrow C(i)$ is induced by the actions of the shuffle $(\mu, \nu)$ on $R^{n+1}$ by permutation of coordinates.

Note that a $C_{2}$-space is just a homotopy-commutative monoid. Furthermore, the usual proof that the loop space of an $H$-space is homotopy-commutative extends to yield the fact that such a loop space is a $C_{n}$-space for every $n$. (It is known that the converse of this fact is false.) We recall from [7] the main theorem on $C_{n}$-spaces, which will be used in the proof of Theorem 1 .

Theorem 0. An associative $H$-space, $Y$, is a $C_{n}$-space if and only if the Hopf fibration for $Y, p_{1}: Y * Y \rightarrow S Y$, extends to a fibration $p_{n}: E_{n}$ $\rightarrow(S Y)_{n}$, where $(S Y)_{n}$ denotes the $n$-fold reduced product space of the suspension of $Y$.

The idea of $C_{n}$-commutativity is somewhat analogous to Stasheff's theory of $A_{n}$-associativity, [5]. Thus the reduced product spaces $(S Y)_{n}$ stand in relation to commutativity much as the projective spaces $X P(n)$ relate to associativity. The proof of the "only if" part of Theorem 0 is inspired by Stasheff's work, and is accomplished by ${ }^{2}$ direct construction in the Dold-Lashof vein. The reverse implication uses the connecting map $r: \Omega(S Y)_{n} \rightarrow Y$ together with the fact that $Y$ is $C_{n}$ in $\Omega(S Y)_{n}$. Here $Y$ is regarded as a subspace of $\Omega(S Y)_{n}$ via the composition $Y \underset{\rightarrow}{\rightarrow} \Omega S Y \subset \Omega(S Y)_{n}$, where $j$ is the usual inclusion. The notion of a subspace being $C_{n}$ in a containing space is a natural extension of the well-known concept of a subspace being homotopy-com mutative in a larger space. mutative in a larger space.
The definition of $C_{n}$-space permits us to state the main theorem ${ }^{\text {of }}$
this note.

THEOREM 1. A space possesses property $P_{n}$ if and only if its 1009 space is a $C_{n}$-space.

The proof of this theorem, which will be outlined below, is based on the following theorem.

THEOREM 2. A monoid, $Y$, is a $C_{n}$-space if and only if the inclusion $i: S Y \rightarrow B_{Y}$ extends to a map $a:(S Y)_{n} \rightarrow B_{Y}$.

The main theorem follows readily from Theorem 2 as follows. Let $X$ be a space and $Y$ a monoid for which there exists a strong $[6]$. homotopy-multiplicative homotopy equivalence $f: Y \rightarrow \Omega X$, as in allu- $^{-}$ Then $f$ induces a homotopy equivalence $g: B_{Y} \rightarrow X$. Let $c$ be the $e$ of the ation map $c: S \Omega X \rightarrow X$. Then $(S f, g)$ is a homotopy equivalence of ${ }^{\circ} S F_{n}$ map $i$ with the map $c$. Now any map $h: S A \rightarrow X$ factors as $c . S h$ 
where $h$ is the adjoint of $h$, and hence $h$ factors up to homotopy through $i$. Now if $\Omega X$ is a $C_{n}$-space, then so is $Y$, and hence $0 \in[i, \ldots, i]$ ( $n$ factors), by Theorem 2. Consequently if $f_{i}: S A_{i} \rightarrow X$, $1 \leqq i \leqq n$, then $0 \in\left[f_{1}, \cdots, f_{n}\right]$, and thus $X$ satisfies property $P_{n}$. Conversely, it follows from Theorem 2.8 of $[3]$ that $0 \in[i, \cdots, i]$ implies that $i$ extends to all of $(S Y)_{n} \rightarrow B_{Y}$.

The proof of Theorem 2 goes as follows. The "if" part is easily obtained from Theorem 0 by taking $p_{n}: E_{n} \rightarrow(S X)_{n}$ to be the fibration induced by $a:(S Y)_{n} \rightarrow B_{Y}$ from $\pi: \mathcal{E}_{\infty} \rightarrow B_{Y}$, the Dold-Lashof universal fibration for $Y$. The converse is the nontrivial implication and is accomplished by using the maps $Q_{i}$ to map $E_{n}$ to $\varepsilon_{n+1}$, the total space of the $(n+1)$ th stage of the Dold-Lashof construction, in fiber-wise fashion, thus inducing a map in the base spaces $(S Y)_{n} \rightarrow Y P(n) \subset B_{Y}$. The details are rather lengthy and will appear elsewhere.
a d a

\section{REFERENCES}

23. M. Arkowitz, The generalized Whitehead product, Pacific J. Math. 12(1962), 7-

2. R. J. Milgram, Iterated loop spaces, Ann. of Math. 84(1966), 386-403.

3. G. J. Porter, Higher order Whitehead products, Topology 3(1965), 123-135.

4. J. Stasheff, On homotopy Abelian H-spaces, Proc. Cambridge Philos Soc. 57 5 , $734-745$.

275-312. Homotopy associativity of H-spaces, Trans. Amer. Math. Soc. 108(1963),

6. M. Sugawara, On the homotopy-commutativity of groups and loop spaces, Mem.

7. Si. Univ. Kyoto Ser. A 33(1960), 257-269.

7. F. D. Williams, Ph.D. thesis, University of Wisconsin, Madison, Wis., 1965.

NEW MExico State University 